\title{
MUJERES EN LA PANTALLA: LA BRUJA FEMINISTA EN CHILLING ADVENTURES OF SABRINA
}

\author{
Laura Álvarez-Trigo \\ Instituto Franklin de la Universidad de Alcalá \\ alvarez.trigo.laura@gmail.com
}

\section{RESUMEN}

La figura de la bruja ha sido en la cultura popular un símbolo comúnmente ligado a la subversión y la independencia de la mujer. Partiendo del estudio de la influencia del Estado y la Iglesia sobre la libertad de las mujeres de Matilda Joslyn Gage, así como del trabajo de Silvia Federici sobre la persecución de las brujas como opresión patriarcal, se propone una exploración de la capacidad de la serie Chilling Adventures of Sabrina (CAOS) para construir la identidad feminista desde la brujería. Sirviéndose también de estudios acerca del rol de las mujeres en los medios, este artículo analiza las estructuras de poder, así como la representación de la sexualidad y de la sororidad en $C A O S$, con el fin de dilucidar si, centrándose en preocupaciones individuales, la serie deja de lado una exploración más profunda de la opresión patriarcal.

Palabras clave: cultura de medios, estudios de género, brujas, feminismo, Sabrina.

WOMEN ON SCREEN: THE FEMINIST WITCH

IN CHILLING ADVENTURES OF SABRINA

\section{Abstract}

The witch figure in popular culture has often been a symbol associated with subversion and women's independence. Based on Matilda Joslyn Gage's exploration of the role of Church and State on the limitation of women's freedom, and Silvia Federici's work on the prosecution of the witches as patriarchal oppression, I investigate the ability of the TV show Chilling Adventures of Sabrina (CAOS) to construct a feminist identity from its protagonist's witchcraft. Also considering previous research on women's representation in media, this article analyzes structures of power, as well as the representation of sexuality and sorority in $C A O S$, with the goal of elucidating if, by focusing on individual concerns, the show casts aside a deeper exploration of patriarchal oppression.

KeYwords: media culture, gender studies, witches, feminism, Sabrina.

DOI: https://doi.org/10.25145/j.clepsydra.2021.20.04

Revista ClepsydRa, 20; febrero 2021, pp. 79-98; ISSN: e-2530-8424 


\section{LA BRUJA COMO ICONO FEMINISTA}

Estudios desde la teoría feminista y el análisis de medios han evidenciado la influencia de los productos mediáticos en nuestro entendimiento del género (ver por ejemplo Bernárdez 2018; Aguirre et al. 2015; Sagro y Plaza 2010). Las estructuras de poder patriarcal se perpetúan a través de la continua representación en los medios de modelos concretos de identidad convertidos en norma. Así, Hollywood, «uno de los fundamentales medios de transmisión de la opresión ideológica de la mujer» (Zecchi 65), crea las «narraciones que utilizamos en la construcción de nuestra propia identidad individual, [...] de nuestra identidad de género» (Sagro y Plaza 25). Es de gran importancia tener en cuenta por tanto que la presencia de mujeres en roles protagonistas ha sido una tendencia generalizada en el cine y la televisión durante los últimos años. Y que, además, su caracterización se opone a menudo a la representación de género tradicional, como podemos ver en Mad Max: Fury Road (2015), Star Wars: The Last Jedi (2016) y Birds of Prey (2020). Sin embargo, como argumenta Asunción Bernárdez, «los modelos convencionales de género siguen siendo los dominantes [...] solo que ese poder se encuentra hoy más oculto, más camuflado, menos visible» (17). Debido a esto es de un gran interés teórico y práctico cuestionar la concepción del sujeto femenino en producciones culturales de éxito. Particularmente en series que han sido señaladas por su contenido feminista como es el caso de Chilling Adventures of Sabrina (CAOS de aquí en adelante).

$C A O S$ ha sido lanzada en tres partes de once, nueve y ocho episodios respectivamente ${ }^{1}$. En el primer episodio conocemos a Sabrina a punto de cumplir los dieciséis años. La joven bruja ha de firmar su nombre en el Libro de la Bestia en un ritual conocido como Bautismo Oscuro si quiere obtener todos sus poderes. No obstante, tiene dudas acerca de los beneficios de entregar su alma al Señor Oscuro (es decir, Lucifer) y en el último momento se niega a hacerlo. En el transcurso de sus aventuras, Sabrina se enfrenta a tradiciones misóginas impuestas por la Iglesia de la Noche y, motivada por Ms. Wardwell, realiza diferentes conjuros en contra de los deseos de su familia y del padre Blackwood, sacerdote de su iglesia y director de la Academia de Artes Ocultas. Al final de la primera parte descubrimos que Ms. Wardwell no es simplemente una bruja al servicio del Señor Oscuro, sino que se trata de Lilith, quien bajo la falsa promesa de llegar a ser reina del infierno sigue las órdenes de Lucifer. Al descubrir la intención del Señor Oscuro de entregar la corona a Sabrina, Lilith se vuelve en su contra y lleva a Lucifer de vuelta al infierno atrapado en el cuerpo de Nick, un hechicero del aquelarre. En la última parte, estrenada en enero de 2020, Sabrina reclama el trono con el objetivo de rescatar a Nick. Mientras tanto, Lucifer logra liberarse de su confinamiento y priva al aquelarre de sus poderes por la traición sufrida, haciendo que no puedan enfrentarse a un grupo de paganos que los amenaza.

${ }^{1}$ A lo largo del artículo se referencian los capítulos indicando la parte y el episodio. Por ejemplo, el episodio 3 de la segunda parte se indicará como P2E3. 
La simbología feminista en la serie es notable por la presencia de las brujas. Esta figura puede concebirse como la personificación de una mujer fuera de los roles tradicionales de género, a quien siempre acompaña la ansiedad masculina causada por el miedo que evoca la feminidad (Gage 1893; Ellis 2017; Thorn 2018). Partiendo de dicho entendimiento, este artículo propone un análisis exhaustivo de los valores feministas contenidos en la serie. Se analiza en consecuencia cómo la serie se posiciona frente a la situación política de los feminismos actuales y sus luchas, situándose en un feminismo considerado aceptable por el mercado de los medios de masas. Se presenta en primer lugar una breve contextualización del rol de la bruja en la cultura popular de las últimas décadas y se ofrece un análisis del origen de su magia en $C A O S$ como un acercamiento a la base de las estructuras de poder. A continuación, se explora la representación de la sexualidad, tradicionalmente ligada a la identidad de la bruja, así como la simbología de la virginidad. Y, finalmente, se analiza la evolución de la posición feminista de los personajes centrándose en los roles de género y la predominancia del individualismo frente a la sororidad.

La asociación entre la brujería y la opresión del sistema patriarcal ha sido extensamente estudiada. Encontramos trabajos de notable interés que analizan esta conexión desde los feminismos de finales del siglo xIx hasta el enfoque marxista sobre la subyugación de las mujeres de Silvia Federici y los análisis más recientes de la cultura popular. Matilda Joslyn Gage expone en Woman, Church and State (1893) que la Iglesia tuvo un papel muy significativo a la hora de definir las diferencias entre hombres y mujeres, vinculando la mujer a algo maligno y negativo, mientras que el hombre representaba lo bueno y divino: «Woman was under an especial curse and man a divinely appointed agent for the enforcement of that curse» (Gage 16). Se llega incluso a cuestionar si las mujeres tienen alma y se establece de esta forma, a través de los dogmas religiosos, la existencia de un sexo superior y un sexo inferior (17). Así, el matrimonio se convertía en un mal necesario para los hombres con el único objetivo de procrear. Sin embargo, la subyugación de las mujeres en términos de su capacidad reproductiva no bastaba para mantener su lado malvado suficientemente controlado, por lo que se establece la tradición del matrimonio desde la perspectiva cristiana. Este nuevo rito necesitaba a la Iglesia para contar con la bendición divina, lo que implicaba preceptos articulados desde la religión como la fidelidad y servidumbre de las mujeres. A través del celibato y de estas narrativas misóginas, la Iglesia y el Estado consiguen controlar la sexualidad femenina, sus cuerpos y sus derechos reproductivos.

Desde el cristianismo, se toma prestado el mito de la bruja para personificar la idea del mal inherente a las mujeres. Aquellas que vivían solas, eran independientes y tenían conocimientos científicos eran consideradas brujas. En el imaginario colectivo, esto se traduce en que las brujas podían tener conocimientos que resultaban peligrosos para la hegemonía existente. La Iglesia prohíbe por tanto cualquier forma de conocimiento a las mujeres, y se muestra "profoundly stirred with indignation at her having through her own wisdom» (Gage 71). Esta asociación entre sabiduría y brujería es importante a la hora de analizar representaciones actuales de la bruja, puesto que sus conocimientos y capacidades serán lo que les brinde la posibilidad de liberarse del yugo del statu quo establecido, como argumenta 
Gage, por la Iglesia y el Estado. En la persecución de las brujas podemos dilucidar, ante todo, un esquema político para situar a las mujeres en una posición inferior a la de los hombres. Como argumenta Silvia Federici en Caliban and the Witch, el resultado de estas políticas "was the enslavement of women to procreation" (89). A través de la imposición del matrimonio y el celibato, el sistema patriarcal podía definir a las mujeres «in terms - mothers, wives, daughters, widows - that hid their status as workers, while giving men free access to women's bodies, their labor» (97). El paradigma que se propone desde la perspectiva de las brujas y de acuerdo con la teoría de Federici se basa en lo cualitativo antes que en lo cuantitativo como resistencia a las limitaciones del sistema capitalista. En clara oposición, muchas de las representaciones actuales de la brujería en la literatura y el cine se enmarcan en una fantasía burguesa. Un claro ejemplo de esto es Harry Potter, ya que «the whole idea that magic might have set rules and could be learnt like a science taught in a school, a private school no less, to be brought under a capitalist social system is a bit of a perversion» (Thorn 8:00).

En lo que respecta a la conexión entre mujer, bruja y maldad, vemos cómo las producciones audiovisuales se van alejando paulatinamente de la representación de la bruja como una mujer fea, malvada y solitaria. Precisamente una de las representaciones de la bruja que más han permeado en el imaginario colectivo durante el siglo xx cuenta con la influencia de Gage, pues fue su yerno, L. Frank Baum, quien escribió The Wonderful Wizard of $\mathrm{Oz}(1900)$ y sus secuelas, inspirando su representación de la mujer y la bruja en la visión política y los textos de Gage. La adaptación cinematográfica de The Wonderful Wizard of $O z$ (1939) tuvo una gran influencia en el símbolo de la bruja moderna y su estética con la aparición de una bruja que utilizaba sus poderes para el bien. Vemos aquí un cambio notable en la forma de representación de la bruja en la cultura popular. En el cine de la primera mitad del siglo se comenzaba a ver a la bruja con unos ojos más compasivos y de forma más positiva, con películas como I Married a Witch (1942) y Bell, Book and Candle (1958). En ellas se presenta a la bruja como una mujer atractiva, joven, rubia, femenina y dispuesta a subyugarse a un hombre si este es su amor verdadero.

En lo que a la televisión se refiere, el personaje de Samantha en Bewitched (1964-1972) nos ofrece una representación también novedosa tanto de la bruja como de la domesticidad de la mujer. La forma en la que Samantha utiliza su magia es molesta para su marido, ya que gracias a sus poderes puede salirse con la suya y, de esta forma, planta la semilla de la liberación. La brujería vuelve a representar un dominio de poder femenino que los hombres (en este caso el marido de Samantha) no pueden poseer ni controlar, pero esto ya no significa que hayan de ser encasilladas en el papel de malvadas. Con estas bases establecidas, en los años 90 comenzamos a encontramos con representaciones divertidas e interesantes de brujas que continúan estando del lado del bien. Algunos de los ejemplos más notables son, sin lugar a dudas, la saga de novelas de Harry Potter (1997-2007), en las que nos encontramos con personajes que podrían considerarse feministas como Hermione Granger y Minerva McGonagall. En lo que respecta a la televisión, se estrenan la muy exitosa serie Charmed (1998-2006) y Sabrina the Teenage Witch (1996-2003). 
El personaje de Sabrina, creado por George Gladir, aparece por primera vez en el número 22 de Archie's Madhouse (1962). Han sido muchas las adaptaciones de Sabrina a la pantalla, siendo la anteriormente mencionada Sabrina the Teenage Witch, con Melissa Joan Hart en el papel protagonista la más exitosa. CAOS no está exclusivamente inspirada en los cómics originales de Archie y en su Sabrina, sino también en otro cómic del mismo nombre escrito por Roberto Aguirre-Sacasa. Una Sabrina satánica y más cruel que la anteriormente conocida aparece en esta versión. La adaptación de Netflix se estrenó en octubre de 2018 y ha sido muy bien recibida tanto por el público como por la crítica. En esta iteración del personaje, Sabrina se da a conocer en el primer episodio como una bruja adolescente y feminista con afición a las películas de horror y el gore. Además, la actriz Kiernan Shipka encarna al personaje, ofreciendo una presentación de belleza blanca normativa.

\section{RELIGIÓN Y ESTRUCTURAS DE PODER}

En el universo de CAOS se presentan diversas estructuras de poder directamente ligadas con la brujería. Por un lado, nos encontramos con la Iglesia de la Noche y, por otro, con el Señor Oscuro como su dios y el origen verdadero del poder de las brujas. Tradicionalmente, lo satánico "provides a path for women who once rebelled against [the] oppressive system because, like all myth, [Satanism] allows us to imagine a different kind of world where women had the power» (Ho 9:03). Sin embargo, la Iglesia que nos presenta CAOS durante la primera temporada se aleja mucho de situar a las mujeres en una posición de poder. La religión satánica se figura como un espejo del cristianismo y de su estructura patriarcal. Se realizan misas regulares oficiadas por un cura varón, existe la excomunión ordenada por los obispos (P1E3), tienen un antipapa y los fieles se confiesan frente al sacerdote de la congregación (P1E8). Es precisamente esta carencia de poder de las mujeres lo que nos plantea interrogantes acerca del símbolo de la bruja como feminista en esta serie.

El personaje de Zelda Spellman, una de las tías y tutora de Sabrina, se define desde el primer momento como una mujer devota y de fe ciega. Vemos a través de ella cómo las brujas se mantienen subyugadas bajo la norma patriarcal de su propia religión. Inicialmente, Zelda es una mujer sumisa, y la única ocasión en la que muestra deseo propio es cuando se entrega sexualmente a Blackwood. Pero esto resulta ser también una subyugación, una entrega al Señor Oscuro por delegación. No obstante, el deseo de Zelda de acercarse al padre Blackwood, así como su respuesta afirmativa a la propuesta de matrimonio, se deben a su anhelo de poder (P2E3), ya que es consciente de que no puede disponer de ese poder dentro del statu quo de su Iglesia si no es a través del matrimonio con un hombre influyente. Esta estructura patriarcal parece reflejar fielmente lo impuesto por el Señor Oscuro. Él es quien otorga su máximo poder a las brujas en cuanto firman el Libro de la Bestia, entregando así su alma y aceptando estar siempre dispuestas a realizar los deseos y órdenes del Señor Oscuro. Dicha entrega incondicional genera dudas en Sabrina, por lo que le cuestiona a Ms. Wardwell/Lilith por qué no es posible para las brujas satánicas tener libertad: 
[Sabrina] But I want both. I want freedom and power.

[Lilith] He'll never give you that. The Dark Lord, the thought of you, of any of us, having both terrifies him.

[S] Why's that?

[L] He's a man, isn't he? (P1E2 24:50).

Merece la pena pararnos a examinar de cerca la respuesta de Lilith. A pesar de estar obedeciendo las órdenes del Señor Oscuro para manipular a Sabrina, admite que la motivación de su Señor viene dada por su naturaleza de hombre. Esta categoría de género masculino se define por tanto como egoísta, con un deseo innato por mantener su poder en detrimento de las mujeres. En el contexto histórico y político en el que se origina la caza de brujas, así como la subyugación de las mujeres, se establece la creencia de que «it's okay for men to access supernatural power through God, but it's not okay for women to access supernatural power through the Devil, and women can't access it through God in Christianity» (Ho 9:35). Así, se asegura que las mujeres no pueden acceder al poder ni desestabilizar la jerarquía patriarcal. Ya que el Seńor Oscuro, en efecto, les otorga poderes sobrenaturales a las brujas, se establece que el orden patriarcal es mantenido a través de la entrega total de sus voluntades. Cualquier cosa que les pida el Seńor Oscuro, en cualquier momento, ellas han de obedecer. Cabe destacar también que se presupone que los hechiceros han de seguir el mismo proceso basándonos en el relato de Ambrose acerca de la petición directa que le ha hecho el Señor Oscuro en el pasado; sin embargo, esta situación de subyugación de los hechiceros no es explorada en la serie del mismo modo que la de las brujas.

El acto de firmar el Libro de la Bestia como condicionante para la obtención de los poderes nos recuerda a otros tratos similares de renuncia a la propia autonomía de las mujeres en la historia. Por ejemplo, a través de firmas de contratos de matrimonio o incluso subyugaciones económicas en la actualidad. En total oposición a dicha renuncia de la autonomía a cambio de poderes superiores, la brujería aparece originalmente como una forma de obtener poder sin esfuerzo ni sacrificio, por lo que su erradicación "was a necessary condition for the capitalist rationalization of work, since magic appeared as an illicit form of power and an instrument to obtain what one wanted without work, that is, a refusal of work in action» (Federici 142). Con la condición necesaria de la firma de su libro, el Señor Oscuro establece una relación de servidumbre opuesta al valor cualitativo de la brujería que, entendida como feminista, reniega de un sistema de intercambio capitalista. Más adelante, la obtención de poder por parte de una mujer en conexión a un contrato matrimonial vuelve a emerger cuando Sabrina finalmente acepta el trono del infierno y afirma que está preparada «to be married to Hell» (P3E8 44:35). CAOS formula por tanto la misma proposición burguesa que encontramos en Harry Potter al establecer la educación formal en la Academia de Artes Ocultas y el sistema extremadamente jerárquico y dogmático que representa la Iglesia de la Noche. Pero, además, reniega de una figuración más feminista de la brujería dada la ausencia de poder innato en las mujeres.

A lo largo de la primera parte, la rebelión de Sabrina y la forma en la que su personaje manifiesta su feminismo no es a través de su cualidad de bruja, sino 
desde su individualidad. Motivada por su ansia de libertad para usar sus poderes, se enfrenta tanto a Blackwood y su aquelarre como al propio Señor Oscuro. Por su parte, Zelda renuncia a su fe motivada en un principio por defender únicamente a su familia. Frente a la rebelión de Sabrina, Blackwood propone al antipapa una reforma de la Iglesia para reafirmar el poder patriarcal: la Iglesia de Judas. Esta nueva Iglesia basa sus preceptos en que las brujas han de servir a los hechiceros igual que Lilith sirve al Señor Oscuro y, por tanto, los hombres han de ser los que estén al mando en la Iglesia y su sociedad. El deseo de Blackwood de deshacerse de las mujeres e impedir su ascenso al poder dentro de la jerarquía de la Iglesia puede entenderse como una aproximación al miedo a la castración, pues este "produce un mecanismo de defensa que o bien tiende a controlar y dominar a la mujer [...] o bien a neutralizarla» (Zecchi 154-155). Tras ver sus planes truncados y huir a Escocia, Blackwood continúa con la búsqueda de una nueva fuente de poder en la que él mismo pueda situarse en lo alto del statu quo. Tras el enfrentamiento de Sabrina con el Señor Oscuro este regresa al infierno atrapado en el cuerpo de un hechicero y en brazos de Lilith. Así, se restablece lo que en apariencia será el nuevo orden matriarcal con Lilith como reina del infierno, quien restaura los poderes de Sabrina diciéndole: «So now my dear you have both power and freedom, and may you never give up either again» (P2E9 54:20). Sin embargo, los planes y el acceso al poder de Lilith se verán de nuevo frustrados a lo largo de la tercera parte de la serie.

Tras estos acontecimientos, Zelda no solamente busca una nueva fuente de poder para el aquelarre, sino una deidad a la que venerar manteniendo así la naturaleza esencialmente religiosa del grupo. Al traicionar a Lucifer, el aquelarre de los Spellman sufre una notable pérdida de magia y salud, por lo que comienzan a rezarle a Lilith. Pero, posteriormente, la reina del infierno se verá abandonada por el propio aquelarre al no serles de utilidad a la hora de recuperar sus poderes y, además, será considerada indigna del trono del infierno al ser vista por los demonios como «Lucifer's whore» (P3E1 48:00). Este desprecio hacia la figura de Lilith resume a la perfección la subyugación y verdadero rechazo que sufre lo abyecto. Para poder salvarse de la ira de Lucifer vuelve, en contra de su voluntad, a ser concubina y madre. A través de su retorno a la sumisión vemos cómo los esfuerzos y deseos de la mujer se ven menospreciados. Paulatinamente, se reafirma la jerarquía propuesta al inicio de la serie. Debemos señalar también que, dentro de este retorno a la jerarquía inicial, durante el intento de recuperar sus poderes a través de Lucifer el aquelarre descubre que el poder del Señor Oscuro no procede de su parte infernal, sino que residen en su naturaleza celestial y su origen como ángel. Esto quiere decir que el poder de la bruja no es meramente satánico y no opera en oposición al orden patriarcal de la Iglesia cristiana. Por el contrario, se origina en él mismo, siendo esta la única y verdadera fuente de poder, es decir, el único orden aceptable. Además, cabe mencionar aquí que Mambo Marie, la única bruja que aparece en la serie como una hechicera cuyos poderes no se originan en Lucifer, es señalada como católica.

Tras el aparente fracaso de Lilith como deidad del aquelarre y por decisión unilateral de Zelda, se convierten en la Orden de Hécate. Esta tradicional diosa griega comúnmente asociada con la magia y la brujería parece encarnar de forma evidente el ideal de poder femenino y a la bruja. Durante el rito de resurrección de 
Hilda, los hechiceros permanecen al margen, mientras las mujeres, tanto brujas como mortales, se cogen de las manos en un círculo, en el centro del cual Zelda recita:

We call on you now Maiden, in your unbounded potential. We call on you Mother, in all your divine power. We call on you Crone, in your arcane wisdom. We are descended of all maidens, mothers, and crones (P3E8 27:00).

En esta invocación observamos una evidente referencia a la esencia femenina de la bruja y a la triple naturaleza de Hécate, equiparable a tres arquetipos tradicionales de la mujer: doncella, madre y anciana. A pesar de lo positivo en el intento de traslación del origen del poder de las brujas de lo masculino-satánico hacia un origen femenino, este cambio no parece motivado por el deseo de romper con la estructura hegemónica opresiva patriarcal, sino por un anhelo de poder y bienestar individual. No es casual que en la simbología contemporánea, la imagen más evidente de Hécate la encontremos en la Estatua de la Libertad. Es precisamente ese deseo de libertad el que lleva a Zelda a recurrir a dicha diosa. En la serie, se presenta la búsqueda de estas diosas como un acto puramente feminista al rechazar cualquier poder originado en una figura masculina. Sin embargo, al analizar de cerca los argumentos y el desarrollo de los personajes, podemos observar que su motivación principal no es la naturaleza femenina de estas figuras mitológicas. Zelda y las demás ven en ellas la posibilidad de obtener mayor poder para sí mismas de forma egoísta. Por tanto, el interés principal no yace en el poder para el aquelarre, sino en la ganancia individual sin importar el bienestar de otras mujeres cuando estas ya no les benefician.

\section{LA SENSUALIDAD Y EL SEXO}

La representación de la sexualidad en $C A O S$ es de central importancia para este estudio por dos motivos principales: debido a la tradicional cosificación de la mujer en la pantalla y a causa de la utilización de la dominación sexual de las mujeres como forma de control de sus cuerpos. En primer lugar, debemos tener en cuenta que la presentación de las mujeres como un objeto erotizado en los medios juega un importante papel en nuestro entendimiento del género. En segundo lugar, como hemos visto en la introducción a través del texto de Gage, la sexualidad y la función reproductiva son un elemento central del control hacia la mujer por parte de la Iglesia y del Estado. Así, se ha establecido «una larga tradición histórica en la que a las mujeres se las calificaba de pasionales, irracionales y dominadas por sus necesidades corporales» (Bernárdez 47). El interés reside por tanto en investigar si la sexualidad se presenta en $C A O S$ como autonomía sobre sus propios cuerpos y liberación o si por el contrario es un elemento inherente a la mujer que se antepone a su capacidad racional. La sexualidad de Sabrina aparece inicialmente de forma poco explícita y se va desarrollando a medida que se convierte en una bruja cada vez más poderosa y se acerca más al Señor Oscuro. La virginidad de Sabrina y de otros personajes se discute en varios momentos de la serie. Esto ocurre por primera vez cuando Sabrina habla con sus tías acerca de su relación con Harvey Kinkle: 
[Sabrina] We very recently took things to the next level.

[Zelda] He hasn't defiled you has he? Witch law forbids novitiates from being anything less than virginal.

[S] [...] Not that it's anyone's business, but no.

[Z] Praise Satan.

[S] However, now that you bring it up, I admit, I have reservations about saving myself for the Dark Lord. Why does he get to decide what I do and don't do with my body?

[Z] Because it is witch law. Covenant (P1E1 42:00).

Este trato de la virginidad como algo que le debe a su Señor refuerza el sistema de poder establecido. La sexualidad de una joven bruja es algo valioso que ha de protegerse solamente para poder ser entregado al Señor Oscuro. Al igual que en el cristianismo la virginidad ha de ser preservada hasta el matrimonio como una forma de controlar la libertad sexual. La cuestión de la virginidad surge de nuevo en tres instancias particulares. La primera ocasión tiene lugar cuando Ms. Wardwell invoca a Las Trece de Greendale (P1E10). Para hacerlo necesita sacrificar a una persona virgen y con este propósito escoge a un chico del instituto de Sabrina. La elección de Ms. Wardwell de sacrificar a un hombre en lugar de a una mujer no parece arbitraria, dado que en diversas ocasiones el personaje se muestra más favorable hacia las brujas que hacia los hechiceros. Por otro lado, este acto nos indica que la valía de la virginidad desde la perspectiva mágica no es exclusiva de las mujeres, aunque no queda claro si los hechiceros han de reservarse para el Seńor Oscuro del mismo modo que lo han de hacer las brujas. La discusión de la virginidad de Sabrina vuelve a tener lugar al llegar la celebración de Lupercalia. Este es el episodio que lidia de forma más directa con la sexualidad de las brujas, así como los mitos y tradiciones que la rodean dentro del folclore de la serie. La festividad de Lupercalia coincide con el día de San Valentín, lo que nos ofrece una metáfora directa entre la sexualidad del mundo de las brujas frente a la idea de amor romántico más asociada a los mortales. El hechicero Nicholas Scratch, con quien Sabrina comienza una relación durante la segunda parte de la temporada, admite esta correlación entre el amor y lo mortal:

You really love him, don't you? That's the only thing I envy about mortals. The way they can give themselves to each other. Witches are aces at passion and lust, but when it comes to love, true love, not so much. [...] Our Dark Lord is a jealous Lord. He wants us to love him and only him (P1E10 11:50).

De nuevo, se evidencia el egoísmo e interés del Señor Oscuro en conservar la servidumbre de sus fieles, así como el statu quo establecido. La diferencia sexual y la dominación del cuerpo son sin duda elementos centrales de la opresión de la mujer, concretamente evidenciados en la persecución de las brujas. Durante el rito de Lupercalia son ellas quienes persiguen a los hechiceros por el bosque hasta alcanzarlos y tener un encuentro sexual que Zelda describe como "a frenzy of orgiastic carnality» (P2E3 4:05). Sin embargo, las parejas son asignadas con anterioridad. Lo que, además de una impuesta heteronormatividad evidente, anula la independencia de las mujeres a la hora de elegir a su pareja sexual. En clara oposición a la conversa- 
ción que Sabrina ha tenido anteriormente con su tía acerca de su vida sexual, en lo que respecta a Lupercalia y dado que Sabrina ya ha firmado su nombre en el Libro de la Bestia, Zelda se muestra mucho más liberal. No solo acepta que Sabrina participe en el ritual, sino que la insta a pensar que puede ser el momento ideal para comenzar su vida sexual.

Desde el enfoque feminista, la subversión de la agencia que aquí se otorga a las mujeres durante «la caza» ofrece una visión de posible liberación sexual. Esta inversión de la subordinación sexual de la mujer, junto con la nula capacidad de decisión acerca de la persona a quien persiguen, resulta ser una fantasía masculina tradicionalmente presente en los medios. A menudo encontramos dicho fenómeno en los cuerpos femeninos cosificados de la publicidad, así como en la construcción de mujeres-muñeca y mujeres-cíborg en el cine, siempre dispuestas y disponibles para los hombres que son sus dueños. Lupercalia ofrece en este mismo sentido un teatro de fantasía masculina. Como argumenta Bernárdez, «en el cine no se muestra la sexualidad femenina, sino un imaginario fantástico de disponibilidad sexual en el que las mujeres no desean nada más que ser deseadas", y este tipo de sexualización «es una forma de cosificación» (72). Sin embargo, también cabe argumentar que, en concordancia con el control de su propio cuerpo asociado a la idea de la bruja, la sexualidad se presenta como intrínsecamente ligada a su poder.

Finalmente, la virginidad como elemento necesario para la magia vuelve a surgir con el grupo de los paganos. Para poder dar vida a su dios, no solamente necesitan sangre de brujas, sino también la sangre de una persona virgen. Theo pierde su virginidad sabiendo esto, de forma que este acto opera como una liberación que lo protege automáticamente de la persecución de los paganos. Al igual que en el primer ejemplo, un hombre es elegido para el sacrificio, en este caso Harvey. Se refuerza así una idea de virginidad ligada a una vulnerabilidad particularmente masculina. Por insistir en la conceptualización de virginidad, al volver atrás en el tiempo y rescatar a Harvey, Sabrina hace que los paganos opten por sacrificar a Ms. Wardwell. Esta elección no ha de pasar desapercibida, pues enfatiza el rol del personaje como solterona puritana, que, a pesar de estar prometida, siguiendo los preceptos de la Iglesia católica más tradicional, se ha mantenido virgen.

A lo largo de la serie encontramos diferentes ejemplos en los que se evidencia cómo las brujas utilizan la magia mediada por la sexualidad para conseguir sus objetivos. El ejemplo más notable ocurre en el segundo episodio, cuando Sabrina pide ayuda a las Weird Sisters ${ }^{2}$ para vengarse de unos compañeros del instituto, jugadores del equipo de fútbol, que han agredido a su amiga Susie (quien pasará a ser conocido como Theo después de su transición). Sabrina y las Weird Sisters utilizan la sexualidad para atraerlos hacia la oscuridad en las minas. A través de una proyección las brujas se desnudan ante ellos y comienzan a besarles. Cuando el hechizo

2 Estas tres hermanas huérfanas (Prudence, Dorcas y Agatha) se hacen llamar Weird Sisters en una evidente referencia a las tres brujas de Macbeth, también conocidas como Weird Sisters o Wayward Sisters. 
desaparece de forma progresiva, resultan ser ellos mismos los que se están besando y Sabrina los amenaza con hacer llegar fotografías de lo ocurrido a todo el instituto. Además, la escopofilia de la mirada masculina, de acuerdo con el marco teórico del psicoanálisis, «no puede separarse del miedo a la castración provocada por la imagen femenina» (Zecchi 155). Y, en efecto, este miedo se hace patente cuando las Weird Sisters aparecen con unos pájaros enjaulados tras haber atacado de nuevo a los chicos en la oscuridad:

[Prudence] We took their boyhoods. Until you release the birds those four boys won't be rising to any occasion. [...] A few weeks of impotence might teach them better manners (P1E2 24:25).

Sabrina no parece muy contenta con este desarrollo de los acontecimientos en lo que se nos muestra como una oposición a los demás hechiceros y brujas, quienes aceptan que han de ser más sexuales como parte de su naturaleza, cosa que Sabrina, siendo medio mortal, parece rechazar. Asimismo, en diversos episodios se muestran repetidas escenas de orgías en las que Sabrina nunca participa y a las que muestra un notable rechazo. En una de estas ocasiones Ambrose, Luke, Nick y las Weird Sisters están en el ático de la casa de los Spellman cuando Sabrina los encuentra. Sabrina actúa tremendamente sorprendida, casi escandalizada, al verlos. Nick la invita a unirse y le ofrece como alternativa irse con ella a su cuarto; Sabrina, en su sorpresa, no es capaz de responderle. En una escena similar, tiempo más tarde, Sabrina descubre a Nick en un encuentro sadomasoquista con dos demonios sexuales y tiene una reacción similar de repulsa y desaprobación. En lo que respecta a las brujas y hechiceros más jóvenes en $C A O S$, como vemos particularmente en las Weird Sisters y en Nick, el poliamor parece ser bastante común. Podemos identificar por tanto una cierta oposición en las brujas a la noción cristiana de sexualidad ligada al puritanismo. Pero, de nuevo, encontramos en su motivación un simple hedonismo, así como una clara falta de perspectiva crítica real hacia la imposición de heteronormatividad y monogamia.

\section{ROLES DE GÉNERO E IDEAL DEL AMOR ROMÁNTICO}

En CAOS la sexualidad aparece ligada a las relaciones románticas y los roles de género. En lo que respecta a estereotipos de género tradicionales, encontramos un grupo de hombres y mujeres en los que estos son particularmente evidentes. La masculinidad se encarna en el personaje del señor Kinkle, el padre de Harvey. Al igual que los jugadores de fútbol del instituto Baxter, el señor Kinkle es un hombre

que tiene aversión a ser percibido como débil. Esta reafirmación de la supremacía asociada a la virilidad tiende a legitimarse con los hombres «poniendo en riesgo sus vidas o las de los demás» (Sagro y Plaza 31), actos que sin duda encontramos en la historia de los hombres de la familia Kinkle: caza, alcoholismo, agresividad y falta de afecto. De acuerdo con la representación tradicional en el cine y la cultura occidental, la masculinidad amenazada «exige en ocasiones respuestas violentas para su defensa» (Sagro y Plaza 31). Siguiendo lo establecido por su padre, Harvey 
comienza a expresar ese miedo a ser percibido como poco viril durante los episodios de la tercera parte. Su temor empieza a hacerse patente al saber que su novia no es virgen. Esta falta de experiencia sexual, además de hacerle vulnerable frente a los paganos, también le hace sentirse débil frente a los chicos más atléticos y fuertes del instituto (P3E3 38:50). El deseo de dominación sobre las mujeres característico de un rol tradicional masculino se representa principalmente en el padre Blackwood. La obsesión por eliminar a las brujas de la jerarquía de la Iglesia de la Noche se refleja en la visión que tiene Ambrose cuando Lilith le lee las cartas. Blackwood le dice: «You are weak brother, for seventy-five years you were imprisoned, made soft by women» (P2E4 52:20). Dado que existe un orden matriarcal en la casa de los Spellman, Blackwood exige a Ambrose que las mate, pues ha de "corregir» la situación, restaurar el orden del hogar, es decir, el orden patriarcal.

La feminidad, de una u otra forma, se hace patente en todos los personajes identificados como mujeres. La mayoría de las brujas adolescentes, especialmente las Weird Sisters, se presentan con un aire de femme fatale, siempre arregladas y maquilladas. También nos encontramos con las mujeres más mayores donde el estereotipo de feminidad se vincula directamente con lo doméstico. El ejemplo más claro de ello es Hilda Spellman, quien actúa de forma maternal y protectora hacia Sabrina y está siempre cocinando para la familia. Lo femenino se asocia así a la domesticidad, los cuidados, la servidumbre. Además, el trabajo de Hilda fuera del hogar da lugar a una relación romántica heteronormativa con su nuevo jefe. Dicha dependencia del amor romántico es uno de los recursos narrativos principales a través de los que la serie presenta los roles de género establecidos. Barbara Zecchi argumenta que «Hollywood promueve las relaciones tradicionales de pareja, la heteronormatividad, ensalza el sacrificio femenino y glorifica a la mujer que elige el amor y la familia frente a la vida profesional» (66). El mito del amor romántico "supone que el amor es algo irracional e involuntario, que el verdadero amor hace sufrir» (Sagro y Plaza 35), lo que generalmente se codifica en violencia hacia la mujer, la cual ha de soportar y perdonar. Cabe evocar también aquí el mito de la existencia del príncipe azul o la media naranja, que implica que dos personas están destinadas la una para la otra (Sagro y Plaza 35). Esta definición del amor romántico a través de sus mitos se ajusta a la presentación de la relación entre Sabrina y Harvey, que incluso se extiende hasta después de su ruptura y la posterior relación de Sabrina con Nick.

El deseo de amor romántico se «ha inscrito en el inconsciente colectivo de los cuerpos socializados como mujeres; el de poner la vida en riesgo, el de saber perdonar y darlo todo por amor, aunque sea la propia vida» (Iturrioz 94). Observamos este deseo de sacrificio por amor en Sabrina en múltiples ocasiones. La mayoría de las decisiones arriesgadas que toma Sabrina están motivadas por sus relaciones románticas. En las dos primeras partes de la serie, las razones de sus actos giran exclusivamente en torno a Harvey, su deseo de que él esté bien y de mantener su relación. Por él Sabrina llega a practicar necromancia, matar a una de sus compañeras de la Academia de las Artes Ocultas y entrar en el limbo. Esta entrega ciega a lo que Sabrina parece codificar como su amor verdadero opera en detrimento de su posición feminista. A menudo antepone el pasar tiempo con su pareja a cualquier otra obligación, ya sea con su familia, con sus estudios, o incluso con aque- 
llos compromisos que ella misma ha adquirido como el trono del infierno. En dos ocasiones diferentes Sabrina le dice a Lilith que en ese momento no puede ir al infierno porque tiene planeado pasar el día con Nick (P3E3 9:10, P3E5 6:30). Si bien esta respuesta puede ser entendida como propia de una adolescente, en ningún momento se expone su comportamiento como reprochable dentro de la narrativa de la serie. Finalmente, a pesar de algún que otro enfado de sus tías o breves reproches e insistencias de Lilith, sus acciones egoístas no tienen ninguna consecuencia negativa. A pesar de que rompe con Nick y al principio parece estar bastante afectada, gracias al hechizo de desvinculación que realiza descubrimos que en el fondo sigue queriendo a Harvey, reforzando así la presentación original del amor entre ambos como el ideal romántico guiado por el destino.

Más allá de las dos relaciones amorosas de Sabrina, las relaciones sexoafectivas heteropatriarcales cobran verdadera relevancia para la crítica feminista a través del matrimonio. Ms. Wardwell, es decir, Lilith, describe el matrimonio (implícitamente entendido como tradicional, heterosexual y probablemente religioso) como una completa pérdida de la libertad por parte de la mujer:

[A] walk down the primrose path towards a woman's destruction. It's nothing less than the complete obliteration of a woman's personhood. It takes everything from her. Her body, her independence, even her soul, and gives nothing in return (P2E5 33:35).

Su afirmación ofrece una visión claramente marcada por un sistema patriarcal opresor, nada diferente a la concepción del matrimonio en el cristianismo. Esto se observa claramente en las relaciones existentes dentro de la Iglesia de la Noche. El padre Blackwood tiene un matrimonio perfectamente tradicional con Constance, quien es profesora en la Academia de Artes Ocultas, permaneciendo de esta forma cercana a su marido y jerárquicamente inferior a él tanto en el matrimonio como en el trabajo. Por otro lado, su embarazo es entendido como medio para asegurar herederos varones. Se trata de nuevo de una reproducción de la opresión por parte de la Iglesia y el Estado explorada por Gage, una forma de controlar a las mujeres a través de su función reproductiva. Las brujas representan tradicionalmente una perversión de las mujeres, cuya función natural e innata era tener hijos (Thorn 11:15); sin embargo, este no es el caso en CAOS. Constance no tiene hijos porque quiera, sino para Blackwood, y cuando tiene complicaciones durante el embarazo admite: «I don't want to lose the babies. [Blackwood] will never forgive me. I don't want to fail him» (P1E7 35:40). Además, los herederos han de ser hombres. Tras una prueba de embarazo, Zelda, quien será la matrona de Constance, informa al padre Blackwood que «the results are promising, the Dark Lord has likely honored you with boys [...] to carry out the Blackwood legacy together. Praise Satan you are truly blessed» (P1E8 50:30). Se mantiene así la idealización de que los hijos varones son más deseables.

Tras la muerte de Constance durante el parto, Blackwood propone matrimonio a Zelda con la intención de ganar control sobre la familia Spellman. De nuevo, asistimos a un intento de restablecer y conservar el orden patriarcal y la subyugación de las mujeres. A través del matrimonio, es decir, de la subyugación a lo domés- 
tico, se asegura la servidumbre y control del cuerpo de las mujeres. Esta atadura a lo doméstico se encarna en el caso del matrimonio entre Blackwood y Zelda en un hechizo al que la somete. Esta situación confirma la visión de Lilith acerca del matrimonio. Bajo el hechizo de Blackwood, Zelda se vuelve complaciente y respeta todos los deseos de su esposo, incluso cambia su forma de vestir. No en vano nos recuerda tanto por la actuación como en lo visual a The Stepford Wives (1975), una ficción en la que todas las mujeres resultan ser robots al servicio de sus maridos.

Finalmente, como parte del ideal de amor romántico no debemos olvidarnos de los celos y la fidelidad. A lo largo de la serie, encontramos dos ejemplos notables de este tipo de comportamiento posesivo hacia las parejas, siempre por parte de la mujer. Este tipo de rivalidad entre mujeres, como veremos en la próxima sección, es un tema muy recurrente en la serie. El más claro ejemplo de celos que vemos en $C A O S$ aparece durante la celebración de Lupercalia (P2E3), cuando el espíritu familiar de Nick, una mujer loba, regresa con intenciones de herir a Nick y a Sabrina por estar juntos. Para solucionar el problema, se pide a Nick que la mate, pero no es capaz, por lo que finalmente es Sabrina quien la asesina en defensa de Nick. Por medio de esta trama, se representa a la mujer de una relación pasada como un ser con intenciones vengativas del que la nueva pareja ha de deshacerse. Posteriormente, Sabrina comienza a sentir celos de la relación de Nick con las Weird Sisters, con quienes él ha salido en el pasado, por lo que le pide a Nick que se aleje de ellas: «I trust you. But I don't trust them» (P2E4 57:50). La responsabilidad de mantener la fidelidad, por tanto, no recae en el hombre, sino en las otras mujeres que se acercan a él como una tentación. De nuevo, se fomenta la competición entre mujeres por el amor y la aprobación de los hombres, perpetuando la idea del amor romántico tradicional.

\section{SORORIDAD FRENTE AL INDIVIDUALISMO}

El conocido eslogan de lo personal es político se volvió famoso gracias al ensayo de Carol Hanisch (1970) a pesar de que ella rechazara la autoría del eslogan. Esta idea pone de manifiesto cómo dentro del ámbito privado los hombres contaban con un gran poder para controlar la vida de las mujeres. Por tanto, el objetivo esencial del feminismo era alcanzar la igualdad también en esos espacios íntimos, más allá de lo público. Esto implica que no pueden existir soluciones individuales a la situación de opresión patriarcal del aquelarre de las brujas en CAOS. En consecuencia, para completar este análisis crítico surge la necesidad de evaluar la relación que las distintas comunidades de mujeres establecen entre ellas para apoyarse en su lucha contra la misoginia tanto en el ámbito público como en el privado.

Como se ha establecido en el apartado anterior en relación con los celos, la rivalidad entre mujeres es bastante ubicua en la serie. Esto se hace patente en un principio en la bienvenida que recibe Sabrina al comenzar a asistir a clases en la Academia de Artes Ocultas, donde sus compañeras comienzan a hacerle lo que parece el equivalente mágico de las novatadas, mucho más siniestro y con riesgo para la vida de ella. Sabrina se muestra escandalizada ante el hecho de que sean brujas las que están haciendo daño a otras brujas y poniendo sus vidas en peligro mediante la 
tradición de los tormentos. Como respuesta a sus quejas, su tía Hilda razona: «You know how cruel girls can be, particularly at that age» (P1E4 4:00), sentando las bases del estereotipo de la mujer como un ser cruel, particularmente hacia otras mujeres.

La actitud negativa hacia las mujeres basada en estereotipos misóginos patriarcales no termina en la visión de las mujeres jóvenes (más notablemente aquellas que son atractivas) como crueles. También se perpetúa un estereotipo negativo acerca de la soltería de las mujeres, especialmente de aquellas que viven solas como «solteronas» (sic). En el primer capítulo Sabrina se encuentra en el cine con su profesora favorita, Ms. Wardwell (antes de ser poseída por Lilith), a quien invita a irse con ella y sus amigos a comentar la película que acaban de ver. Frente a este intercambio, una de sus amigas cuestiona a Sabrina haberle ofrecido a la profesora que se fuese con ellos, a lo que ella responde: «I feel bad for her, living in that house... all alone» (P1E1 3:25). A pesar de que la profesora le simpatiza, Sabrina hace un juicio de valor mediado por estereotipos negativos de género. Incluso la propia Lilith posteriormente, en el cuerpo de Ms. Wardwell, se lamenta: «[The Dark Lord] wishes I maintain this humiliating pretense that I'm some unattractive, unhappy spinster schoolmarm» (P2E3 8:10). Lilith, quien no duda en criticar el matrimonio como una subyugación de la mujer y una decisión que solo ha de traerle desgracias, asume irónicamente que Ms. Wardwell, por soltera y solitaria, es infeliz.

Continuando el análisis de las relaciones establecidas entre mujeres, nos encontramos con que los lazos más vinculantes y presentes en la serie son entre hermanas. Los dos conjuntos de hermanas más predominantes son las tías de Sabrina y las Weird Sisters. Ambos grupos parecen convivir en un estado jerárquico donde todas las decisiones son tomadas por Zelda y Prudence respectivamente. La relación fraternal entre Hilda y Zelda se presenta como tortuosa. Zelda incluso llega a matar a Hilda (aunque resucitándola después) por darle consejos a Sabrina con los que ella discrepa (P1E2). Por su lado, Prudence, tras descubrir que sus hermanas Dorcas y Agatha han realizado un conjuro sin su permiso, les prohíbe volver a hacerlo sin su consentimiento explícito (P1E8) y, al igual que en el caso de las Spellman, esto culmina con el asesinato y posterior resurrección de una de las hermanas como venganza. El motivo por el que todas ellas se encuentran en estas relaciones jerárquicas tan evidentes no se esclarece en ningún momento. Por un lado, puede entenderse como la crueldad innata en las mujeres que la propia narrativa pone de manifiesto y, por otro, como la reproducción del sistema jerárquico en el que habitan como única forma de relación que conocen.

Si entendemos estas dinámicas como parte de la crueldad hacia otras mujeres inherente a la naturaleza femenina, se evidencia de nuevo la conexión de estos comportamientos con los celos. Y a su vez, los celos como aspecto central de la mecánica de las relaciones que establecen las mujeres. El vínculo entre las hermanas Spellman se ve en diversas ocasiones afectado por las relaciones románticas, debido a la envidia que estas generan en la que permanece soltera. A lo largo de la tercera temporada, en la que Dr. Cee le pide matrimonio a Hilda, la relación entre ella y su hermana se ve gravemente perjudicada tras un periodo de aparente mejoría motivado por la separación de Zelda y Blackwood. Ante la noticia del compromiso de Hilda, Zelda afirma: «I'm not mad that Sabrina and you are abandoning 
me» (P3E4 20:45). Esta reacción pone de manifiesto el profundo resentimiento que siente por su hermana. La decisión de prometerse de Hilda es entendida como excluyente a seguir pasando tiempo con su hermana y su sobrina. La complicación que supone presentar la relación romántica de Hilda como una amenaza directa hacia su hermana se refuerza en la trama de la novela que Hilda escribe bajo pseudónimo. El resumen de la novela, que Zelda lee, habla de una bruja y su amante, que son «terrorized by the witch's loveless spinster hag of a sister» (P3E3 19:40). De nuevo, observamos en la descripción de la mujer solterona y sin amor unas fuertes connotaciones patriarcales asociadas a la incapacidad de encontrar un hombre con el que mantener una relación heteronormativa tradicional.

La rivalidad entre mujeres no se limita exclusivamente al ámbito amoroso y familiar, sino que también encontramos ejemplos en el laboral. En la Academia de Artes Ocultas, cuando Zelda entra a sustituir a Constance, se establece una inmediata rivalidad entre ella y otra de las profesoras, Shirley Jackson ${ }^{3}$. En este caso la competición es sin duda por el poder. Dado que el poder al que las mujeres pueden acceder es siempre limitado, Shirley no puede permitirse tener una rival con quien el padre Blackwood tenga un trato de favor. Con el objetivo de eliminar la competencia, Shirley le dice a la fallecida Constance que Zelda la mató, robó a sus bebés y ahora va a robar a su marido (P2E5 19:40), haciendo que su fantasma la persiga. Posteriormente, cuando Hilda la confronta acerca de sus acciones, Shirley confiesa que efectivamente lo hace porque no quiere que una Spellman ascienda más que ella, a lo que Hilda le responde: «So, in actuality, you are just a petty and jealous bitch» (P2E5 29:00). De nuevo, la reacción de Hilda reitera estereotipos misóginos, como la mezquindad y los celos. La defensa que hace de Zelda, no debemos olvidarnos, no la hace motivada por ningún sentimiento de justicia, sino simplemente porque es familia.

Cuando el padre Blackwood pretende reformar la Iglesia de la Noche bajo preceptos aún más misóginos que los hasta entonces establecidos, Zelda habla con Prudence para que le dé la espalda a su Iglesia. Pero solo una vez que Blackwood envenena a todo el aquelarre, vemos cómo las mujeres comienzan a unirse en contra del símbolo del patriarcado que representa el padre Blackwood y a protegerse unas a otras. Salvo contadas excepciones, no existe un deseo ni motivación real de las mujeres y brujas en $C A O S$ de ayudarse entre ellas para cambiar la situación de todas, a no ser que la mujer a la que estén ayudando sea una amiga o un familiar directo. Incluso cuando Sabrina salva a Prudence durante el Festín de los Festines de ser canibalizada por el resto de las brujas, Prudence sabe que Sabrina no lo ha hecho por ayudarla ni porque sean amigas, sino por ella misma. Le echa en cara que, en efecto, «the only occasions when you dare speak to me or my sisters is when you need something from us» (P2E8 5:05). Este egoísmo denota un claro trasfondo individualista en el personaje de Sabrina y, por extensión, en la serie. A través de la representación

\footnotetext{
${ }^{3}$ Se da a entender a través de la apariencia física del personaje que se trata en efecto de la autora Shirley Jackson, aunque no se hace ninguna mención directa.
} 
de las relaciones entre mujeres, la narrativa nos presenta un feminismo que se aleja de la sororidad y de la búsqueda de un cambio sistémico.

Finalmente, dentro de la carencia generalizada de perspectiva comunitaria, debemos examinar el comportamiento de los diferentes grupos y aquelarres frente a la amenaza que suponen los paganos, así como la fuga de Lucifer. Con sus poderes debilitados, el aquelarre de las Spellman se ve en la necesidad de pedir ayuda a las conocidas como «brujas del cerco». Hechiceras que viven por su cuenta fuera de cualquier aquelarre o comunidad. Estas acceden a ayudar a regañadientes gracias a un reproche de Mambo Marie, quien les recuerda que no han de pelearse entre ellas porque "that is what men do. But we are women» (P3E6 2:30); y a que Zelda les hace ver que su propia seguridad y modo de vida también está en juego. El hecho de que las demás brujas obedezcan y muestren interés en Mambo Marie despierta en Zelda cierta envidia que motiva una nueva rivalidad. Como Prudence le hace ver, Zelda rechaza a Mambo Marie porque no comprende de dónde vienen sus poderes, y corre el riesgo de que su posición en la jerarquía del aquelarre pueda verse amenazada por la presencia de esta nueva bruja (P3E6 25:15). De nuevo, la posición de poder a la que puede acceder una mujer parece ser imposible de compartir. Se refuerza una vez más la idea de que la competencia entre las mujeres es necesaria, y la inversión del statu quo se centra en el individuo y no en el colectivo. Por ańadidura, en medio de toda esta agitación, Lilith también se encuentra desamparada y pide asilo a Zelda en la academia, pero es rechazada. Zelda dice que ha de encontrar otro lugar para esconderse, puesto que rezarle no les ha traído ningún beneficio (P3E6 18:10). En este repudio se evidencia de nuevo el egoísmo de las brujas y de Zelda en particular. $\mathrm{Al}$ no haber recibido favores ni ganancias siéndole fiel a Lilith, esta no merece su compasión ni ayuda en momentos de extrema necesidad.

\section{CONCLUSIONES}

A través de la Iglesia de la Noche y los preceptos del Señor Oscuro las brujas se encuentran subyugadas al igual que el resto de las mujeres. La serie no nos ofrece una visión de lo abyecto a través del feminismo de la bruja, sino que nos aporta una réplica de las opresiones implantadas tradicionalmente por el patriarcado sobre las mujeres, sus cuerpos y sus libertades. La imagen de la brujería que nos encontramos en $C A O S$ establece un claro contraste con una de las más notables concepciones tradicionales de esta, conectada a ontologías matriarcales vinculadas a la naturaleza. Aunque no debemos pasar por alto la inclinación final de Zelda de realizar un acercamiento a una diosa femenina, tradicionalmente asociada a la brujería y al poder de la naturaleza. Sin embargo, como hemos visto a través del análisis de la forma en la que la serie trata diferentes aspectos que consideramos intrínsecamente ligados al feminismo, este cambio se origina en un marcado individualismo. Zelda desea mantener su poder dentro del aquelarre y la primera petición que realiza a la diosa Hécate es que resucite a su hermana, quien no debería ser de más valor que otras brujas que han sido también asesinadas ese mismo día y a quienes no parece tener intención de resucitar. 
En lo que respecta a la representación de la sexualidad desde un punto de vista rompedor con los cánones heteropatriarcales, la serie también se mantiene en un espacio seguro donde sus transgresiones no son particularmente radicales. La representación de una sexualidad más afín a la libertad fuera del sistema y la jerarquía establecida debería encontrarse principalmente en la bruja protagonista. Pero descubrimos que Sabrina a menudo toma decisiones motivada por su deseo de amor romántico heteronormativo. Asimismo, cabe también recordar que la autonomía que se da a las mujeres en términos de sexualidad es más superficial que real. De esta forma, el personaje de Sabrina se mantiene virginal, distanciado de la más liberada Prudence, a quien se nos presenta como manipuladora y promiscua. En la misma línea que ocurre con la sexualidad y las relaciones románticas, los roles de género en raras ocasiones resultan particularmente rompedores. Esto lo podemos observar con mayor nitidez en las relaciones que las mujeres establecen entre sí a lo largo de la temporada. El personaje de Ms. Wardwell es el que mejor ilustra esta tendencia de perpetuación de estereotipos negativos y de roles tradicionales desde su caracterización como solterona infeliz y solitaria. Este estereotipo no solo no es cuestionado por ninguna otra mujer de la serie, sino que son ellas mismas quienes lo perpetúan.

La serie evidencia la sociedad patriarcal a la que las brujas han de enfrentarse en los comportamientos masculinos. Esta producción se ha enmarcado como feminista gracias a su trama y mediante una clara oposición y enfrentamiento beligerante de los personajes principales a los hombres del statu quo. Así se nos presenta una visión maniquea y simplificada del problema del patriarcado, donde los hombres que se encuentran en posiciones de poder actúan abiertamente con intención de subyugar a las mujeres. De esta forma, se simplifica la lucha feminista, pues no parece haber nada sistémico ni más sutil que cuestionar. La opresión se personifica en hombres que narran de viva voz su intención de quitar poder a las mujeres, pero nunca en la visión misógina de la caracterización de mujeres como celosas y vengativas por naturaleza. Esto se manifiesta también en el hecho de que los comportamientos machistas no son solo perpetrados por hombres, sino que también nos encontramos a mujeres reforzando estereotipos patriarcales. Entre estos elementos misóginos cabe destacar la representación de las solteronas, la competitividad entre mujeres y los celos. Además, las estrategias de lucha no construyen la búsqueda de una sociedad igualitaria por razones éticas, sino que la narrativa conduce a ponerse de parte de Sabrina como individuo. Por tanto, la serie conserva la mentalidad predominantemente sexista inherente al sistema.

La conclusión innegable de la falta de sororidad entre los personajes femeninos es que la visión del feminismo ofrecida por CAOS es claramente individualista. El giro hacia Hécate, dada su personificación en la Estatua de la Libertad, no puede ser pasado por alto. Se hace a través de ella una clara referencia, no a la búsqueda de una fuente de poder en lo femenino, sino a un retorno al individualismo que debería haber estado en lo satánico. En la línea de una creciente tendencia actual dentro del movimiento feminista en la que prima el triunfo individual frente al social, Sabrina desea tener poder y libertad para ella misma, no para las mujeres/ brujas como colectivo. Sabrina a menudo recurre a soluciones individualistas y la propia narrativa presenta una marcada reticencia a la exploración de una oposición 
más directa, de lo que sería realmente la bruja, lo satánico. Se entiende que a través de sus actos individuales es posible, y probable, que la situación general mejore, pero no es en ningún momento lo que motiva al personaje. Este énfasis en lo individual es propio de la cultura occidental, opuesta a una ontología relacional, colaborativa, más propia del arquetipo de la bruja.

Es necesario por tanto aportar una mirada crítica hacia aquellas producciones mediáticas que se clasifican como feministas y se muestran como tales desde una perspectiva muy superficial. Sin duda, CAOS nos presenta un argumento que abre las puertas a la heroína para que luche activamente por su propia historia sin ser relegada a un segundo plano por el relato de un hombre. Pero esto ha de hacerlo en una época en la que el feminismo mediático exitoso parece centrarse principalmente en preceptos superficiales que han de encajar con la imagen de mujer deseable y belleza clásica, beligerante contra el patriarcado pero nunca desde lo abyecto. Haciendo un análisis de la muñeca Barbie en la actualidad, de nuevo Bernárdez argumenta que el ideal de feminidad perfecta ya no existe, sino que nos encontramos con «una mezcla imposible de sexualización, independencia, y empoderamiento» (65). Este empoderamiento individualista, por tanto, nos separa de la concepción de la bruja que Federici identifica como verdaderamente fuera del sistema patriarcal, como un sistema primitivo de acumulación.

Enviado: 25 de marzo de 2020; ACEptado: 5 de octubre de 2020 


\section{BIBLIOGRAFÍA}

Aguirre Sacasa, Roberto. Chilling Adventures of Sabrina. Netflix, 2018-2019.

Bernárdez Rodal, Asunción. Soft Power: Heroinas y muñecas en la cultura mediática. Madrid: Editorial Fundamentos, 2018.

Ellis, Lindsay. «Loose Canon: The Wicked Witch of the West», en Lindsay Ellis, 22 de marzo, 2017 (https://www.youtube.com/watch?v=xiZB4WgjJmA).

Federici, Silvia. Caliban and the Witch: Women, the Body and Primitive Accumulation. Brooklyn: Autonomedia, 2004.

Gage, Matilda Joslyn. Woman, Church and State. Nueva York: The Truth Seeker Company, 1893 (http://www.public-library.uk/ebooks/107/39.pdf).

Hanisch, Carol. «The personal is political», 1969.

Ho, Soleil. «Popaganda: The Devil You Know», en Bitch, 1 de noviembre 2018 (https://www.bitchmedia.org/article/popaganda-devil-you-know).

Iturrioz, Josebe. «Buffy cazavampiros: Feministas, lesbianas y brujas», en Aguirre, Katixa et al. (ed.). Virgenes catódicas. Putas recalcitrantes. Tafalla: Txalaparta, 2015, pp. 63-125.

Keller, Douglas. Media Culture: Cultural Studies, Identity and Politics between the Modern and the Post-modern. Nueva York: Routledge, 1995.

Kristeva, Julia. Powers of Horror: An Essay on Abjection. New York: Colombia University Press, 1982.

SAgro, Pedro y Plaza, Juan Francisco. La representación de la mujeres en el cine y la televisión contemporáneos. Barcelona: Laertes editorial, 2010.

Thorn, Oliver. «Witchcraft, Gender, \& Marxism», en Philosophy Tube, 26 de octubre, 2018 (https:// www.youtube.com/watch?v=tmk47kh7fiE).

Zeсchi, Barbara. La Pantalla Sexuada. Madrid: Cátedra, 2014. 